\title{
Design and Validation of a General Purpose Robotic Testing System for Musculoskeletal Applications
}

\author{
Lawrence D. Noble \\ Cleveland Clinic \\ Robb W. Colbrunn \\ Cleveland Clinic \\ Dong-Gil Lee \\ Cleveland Clinic
}

Antonie J. van den Bogert

Cleveland State University, a.vandenbogert@csuohio.edu

Brian L. Davis

Cleveland Clinic

Follow this and additional works at: https://engagedscholarship.csuohio.edu/enme_facpub

Part of the Biomechanical Engineering Commons

How does access to this work benefit you? Let us know!

Publisher's Statement

The final publication is available at Springer via http://dx.doi.org/10.1115/1.4000851

\section{Original Citation}

Noble, L. D., Colbrunn, R. W., Lee, D., van den Bogert, A. J., and Davis, B. L., 2010, "Design and Validation of a General Purpose Robotic Testing System for Musculoskeletal Applications," Journal of Biomechanical Engineering, 132(2) pp. 025001-025001.

This Article is brought to you for free and open access by the Mechanical Engineering Department at EngagedScholarship@CSU. It has been accepted for inclusion in Mechanical Engineering Faculty Publications by an authorized administrator of EngagedScholarship@CSU. For more information, please contact library.es@csuohio.edu. 


\section{Design and Validation of a General Purpose Robotic Testing System for Musculoskeletal Applications}

\section{Lawrence D. Noble, Jr.}

\section{Robb W. Colbrunn}

Department of Biomedical Engineering, Lerner Research Institute, and

Orthopaedic and Rheumatologic Research Center, Cleveland Clinic,

Cleveland, OH 44195

\author{
Dong-Gil Lee \\ Department of Biomedical Engineering, \\ Lerner Research Institute, and \\ Orthopaedic and Rheumatologic Research Center, \\ Cleveland Clinic, \\ Cleveland, OH 44195; \\ Department of Orthopaedics and Sports Medicine, \\ University of Washington, \\ Seattle, WA 98195
}

\section{Antonie J. van den Bogert}

\author{
Brian L. Davis ${ }^{1}$ \\ e-mail: davisb3@ccf.org
}

\author{
Department of Biomedical Engineering, \\ Lerner Research Institute, and \\ Orthopaedic and Rheumatologic Research Center, \\ Cleveland Clinic, \\ Cleveland, OH 44195
}

\begin{abstract}
Orthopaedic research on in vitro forces applied to bones, tendons, and ligaments during joint loading has been difficult to perform because of limitations with existing robotic simulators in applying full-physiological loading to the joint under investigation in real time. The objectives of the current work are as follows: (1) describe the design of a musculoskeletal simulator developed to support in vitro testing of cadaveric joint systems, (2) provide component and system-level validation results, and (3) demonstrate the simulator's usefulness for specific applications of the footankle complex and knee. The musculoskeletal simulator allows researchers to simulate a variety of loading conditions on cadaver joints via motorized actuators that simulate muscle forces while simultaneously contacting the joint with an external load applied by a specialized robot. Multiple foot and knee studies have been completed at the Cleveland Clinic to demonstrate the simulator's capabilities. Using a variety of general-use components, experiments can be designed to test other musculoskeletal joints as well
\end{abstract}

\footnotetext{
${ }^{1}$ Corresponding author.
}

(e.g., hip, shoulder, facet joints of the spine). The accuracy of the tendon actuators to generate a target force profile during simulated walking was found to be highly variable and dependent on stance position. Repeatability (the ability of the system to generate the same tendon forces when the same experimental conditions are repeated) results showed that repeat forces were within the measurement accuracy of the system. It was determined that synchronization system accuracy was $6.7 \pm 2.0 \mathrm{~ms}$ and was based on timing measurements from the robot and tendon actuators. The positioning error of the robot ranged from $10 \mu \mathrm{m}$ to $359 \mu \mathrm{m}$, depending on measurement condition (e.g., loaded or unloaded, quasistatic or dynamic motion, centralized movements or extremes of travel, maximum value, or root-mean-square, and $x$-, $y$ or z-axis motion). Algorithms and methods for controlling specimen interactions with the robot (with and without muscle forces) to duplicate physiological loading of the joints through iterative pseudo-fuzzy logic and real-time hybrid control are described. Results from the tests of the musculoskeletal simulator have demonstrated that the speed and accuracy of the components, the synchronization timing, the force and position control methods, and the system software can adequately replicate the biomechanics of human motion required to conduct meaningful cadaveric joint investigations.

Keywords: orthopaedic biomechanics, foot and ankle, knee, robotics, instrumentation, simulation, actuators

\section{Introduction}

The fundamental understanding of strain and stress within bone and soft tissue during various loading conditions is of great importance to researchers of degenerative diseases, injury prevention, and rehabilitation. In vivo and in vitro studies as well as computational modeling have helped investigators gain valuable insights into the strains and stresses developed within the joint in response to loading, but each technique has some inherent limitation. Human in vivo studies of load-induced bone strains, as might be experienced during exercise, are difficult to conduct because of the nature of the invasive surgery required to implant strain gauges and the failure of bonding techniques between strain gauges and bone during exercise $[1,2]$. In vivo studies designed to measure tissue breakdown using strain gauges could provide significant insight to progressive diseases such as diabetes. However, for ethical and scientific reasons, this is not practical. Furthermore, from a scientific standpoint, obtaining accurate, repeatable in vivo results during long-term joint loading sessions would be difficult because of variability of responses from one trial to another, even within the same subject. Computational models to predict internal tissue loads based on external motion and applied loads require accurate data on tissue geometry and material properties. Reliability of these models is still problematic for mechanically complex systems such as the knee or foot, wherein soft tissue plays an important role $[3,4]$. In contrast, in vitro testing with cadavers under simulated loading conditions can complement these other techniques and offers additional advantages. Musculoskeletal simulators and loading devices have been developed [5-10] to study the lower extremities. By reproducing varying degrees of the target kinematics and kinetics in vitro, investigators have acquired meaningful and clinically relevant data. Although these previous simulators have yielded new insight into the biomechanics of those particular joints, our general-purpose musculoskeletal simulator can support a wider range of investigations because of the following capabilities:

1. simulating loading conditions on multiple joints (knee, hip, wrist, shoulder, etc.)

2. simulating various loading conditions beyond walking (running, jumping, etc.)

3. scaled velocities that simulate real-time (or near real-time) dynamics 


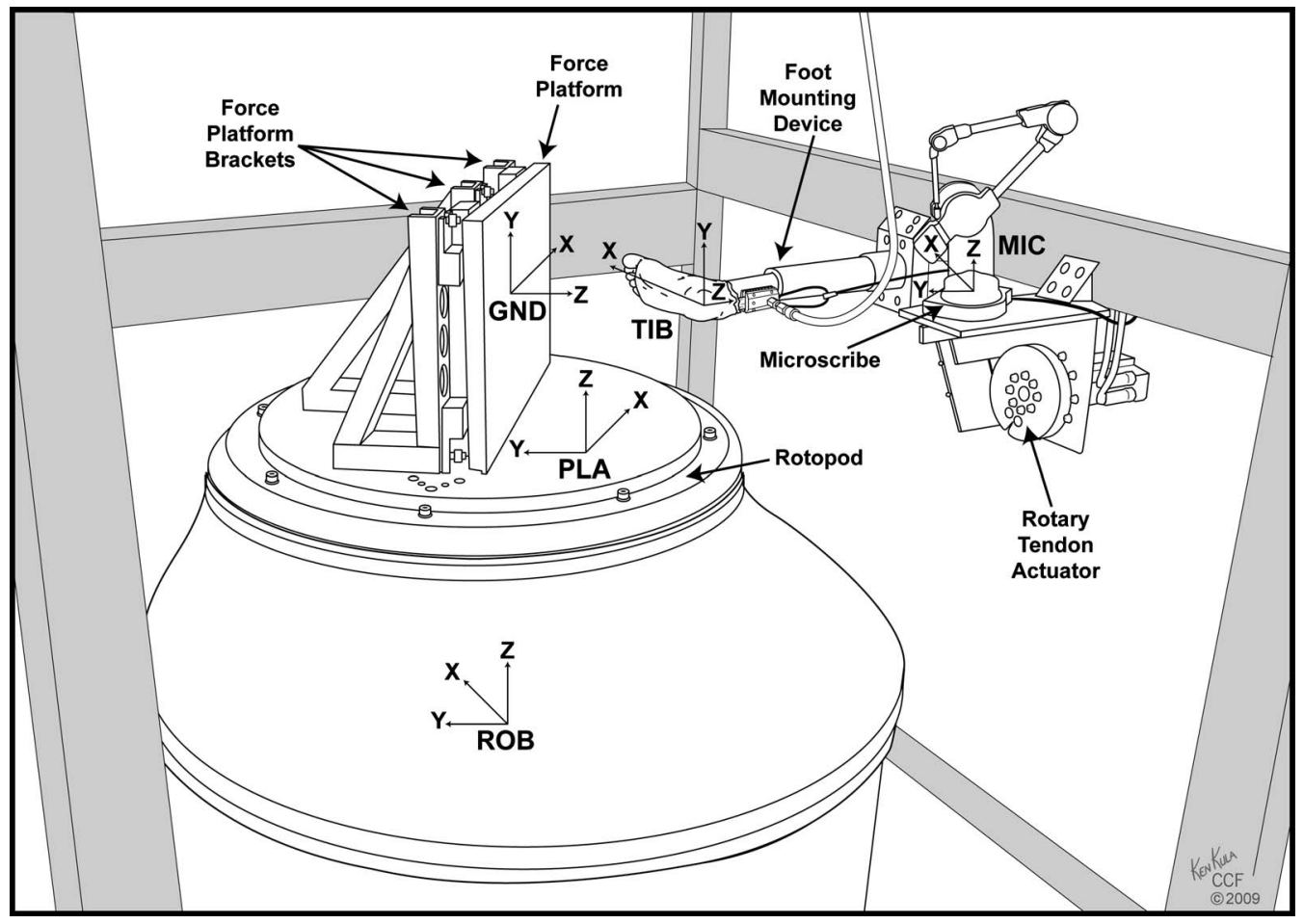

Fig. 1 Simplified illustration of the musculoskeletal simulator, as it would be configured for a foot study. The various coordinate systems shown illustrate the necessary mathematical transformations required to achieve motion of the force platform against the foot to simulate gait (GND: force plate; MIC: MicroScribe; PLA: rotopod platform; ROB: rotopod base; TIB: tibia). Reprinted with permission, Cleveland Clinic Center for Medical Art \& Photography @2009. All Rights Reserved.

4. simulating loading conditions in all six degrees of freedom (DOF) as compared with simple planar motion

5. simulating full- or near full-physiological loading (internal muscle forces and external forces) of the joint

The musculoskeletal simulator has been developed to simulate a large spectrum of loading conditions for essentially any joint of interest through coordinated control of the external loading device (rotopod) and tendon actuators (servomotors). Knowledge of the specimen location and orientation with respect to the external loading device is provided using a spatial digitizer. The musculoskeletal simulator uses this knowledge to form kinetic and/or kinematic inputs to drive the devices based on the target loading conditions. To control these loading conditions, the musculoskeletal simulator can be configured to employ either (1) position control, (2) iterative optimization (affecting kinetic and kinematic trajectories), or (3) real-time proportional-integral-derivative (PID) force feedback control.

The objectives of the current work are as follows: (1) describe the design of a musculoskeletal simulator developed to support in vitro testing of cadaveric joint systems, (2) provide component and system-level validation results, and (3) demonstrate the simulator's usefulness for specific applications of the foot-ankle complex and knee.

\section{Materials and Methods}

\subsection{Component Design}

2.1.1 Design Overview. The major components of the musculoskeletal simulator (Fig. 1) are the tendon actuators, rotopod, MicroScribe, external sensor data acquisition system, and external loading sensor. The type of external loading sensors used is based on the particular joint under investigation. The foot application used a six-axis force platform to measure forces and moments, whereas a six-axis load cell was used in the knee studies. Additional components of the musculoskeletal simulator include the specimen mounting device, tendon load cells, tendon freeze clamps, knee flexion fixture, and application software.

2.1.2 Tendon Actuators. Three different tendon actuators have been developed to meet the unique demands of different muscle groups in the leg. It was assumed that the most rigorous exercise tested would be running and that the Achilles actuator would be the most demanding. We estimated, using gastrocnemius muscle kinematic data from Cavanagh [11], that the peak tensile force would be $2300 \mathrm{~N}$, velocity of $0.54 \mathrm{~m} / \mathrm{s}$, and acceleration of $56 \mathrm{~m} / \mathrm{s}^{2}$. Actuators are attached to tendons through pulley/cable systems that terminate at the freeze clamps, which are affixed to the tendons (Fig. 2). The rotary actuator consists of a Baldor (Fort Smith, AR) model BSM80N-275AE servomotor, a harmonic drive

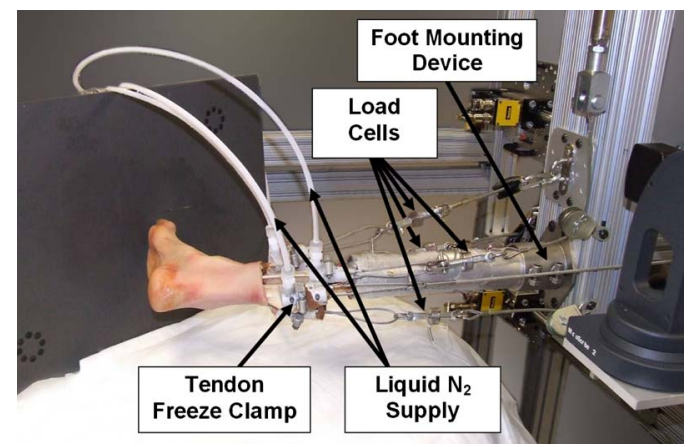

Fig. 2 Musculoskeletal simulator, demonstrating cadaver foot mounting and attachment of five tendons to the actuators through freeze clamps, cables, and pulleys 


\begin{tabular}{lc}
\hline \hline Feature & Value \\
\hline Drive reduction ratio & $50: 1$ \\
Peak static force $(\mathrm{N})$ & 6110 \\
Continuous force $(\mathrm{N})$ & 1880 \\
Maximum velocity $(\mathrm{m} / \mathrm{s})$ & 0.40 \\
Maximum acceleration $\left(\mathrm{m} / \mathrm{s}^{2}\right)$ & 120 \\
\hline \hline
\end{tabular}

system (Hauppauge, NY) model CSG-40-50 harmonic drive, and a $175 \mathrm{~mm}$ diameter pulley (Table 1). This actuator was selected because it can exceed the force of the Achilles tendon during rigorous exercise. The velocity and acceleration capabilities of the actuator suggest that it can perform simulations of near real-time running. Since it incorporates a pulley system, there are practically no limitations regarding tendon stroke, making this actuator suitable for simulating the action of many different musculoskeletal systems. The linear actuators are Parker Hannifin Corp. (Cleveland, OH) ET50-Series electric actuators with SM233A servomotors (Table 2). Two different varieties of linear actuators have been developed. One design provides a $50-\mathrm{mm}$ stroke and the other a $100-\mathrm{mm}$ stroke. The 50-mm stroke design was selected because the muscles used in the foot during walking would not exceed this range. The $100-\mathrm{mm}$ stroke was selected for some future application that might need an extended stroke. The peak force is sufficient for the other muscles, and the velocity and acceleration parameters indicate that running simulations at half speeds are possible (note that acceleration scales by one-fourth when speed is scaled by one-half).

2.1.3 Rotopod. The R2000 rotopod, developed by Parallel Robotic Systems Corp. (Hampton, NH), is a 6DOF robot (Table 3). The rotopod is similar to a standard hexapod robot, but, due to the unique mounting configuration of the six actuators on a circular path, it is additionally capable of rotating a payload of $\pm 720 \mathrm{deg}$ about the Z-axis of the rotopod base coordinate system (ROB) (Fig. 1). The high load capacity of the rotopod makes it possible to provide full-physiological loading simulations, including running loads [12]. However, the velocity capabilities suggest run-

Table 2 Linear tendon actuator characteristics

\begin{tabular}{lc}
\hline \hline Feature & Value \\
\hline Peak static force $(\mathrm{N})$ & 1450 \\
Continuous force $(\mathrm{N})$ & 560 \\
Maximum velocity $(\mathrm{m} / \mathrm{s})$ & 1.0 \\
Maximum acceleration $\left(\mathrm{m} / \mathrm{s}^{2}\right)$ & 14 \\
\hline \hline
\end{tabular}

Table 3 Rotopod specifications

\begin{tabular}{lc}
\hline \hline Feature & Value \\
\hline Platform size (diameter) $(\mathrm{mm})$ & 780 \\
Load capacity $(\mathrm{N})$ & 2000 \\
Torque capacity $(\mathrm{N} \mathrm{m})$ & 1000 \\
Payload capacity $(\mathrm{kg})$ & 227 \\
Translational velocity $(\mathrm{mm} / \mathrm{s})$ & 100 \\
Angular velocity $(\mathrm{deg} / \mathrm{s})$ & 120 \\
Static accuracy $(\mu \mathrm{m})$ & \pm 50 \\
Repeatability $(\mu \mathrm{m})$ & 25 \\
$X$-axis range of motion $(\mathrm{mm})$ & \pm 110 \\
$Y$-axis range of motion $(\mathrm{mm})$ & \pm 110 \\
$Z$-axis range of motion $(\mathrm{mm})$ & \pm 93 \\
Roll range of motion $(\mathrm{deg})$ & \pm 13 \\
Pitch range of motion $(\mathrm{deg})$ & $+12,-19$ \\
Yaw range of motion $(\mathrm{deg})$ & \pm 720 \\
\hline \hline
\end{tabular}

\begin{tabular}{lc}
\hline \hline Feature & Value \\
\hline Workspace (cm sphere) & 168 \\
Resolution (mm) & 0.13 \\
Accuracy (100 point ANSI sphere) (mm) & 0.43 \\
\hline \hline
\end{tabular}

ANSI: American National Standards Institute.

ning simulations must be time scaled. The motion path and corresponding velocities required of the robot for simulating running will exceed the translational and rotational velocity capabilities of the robot. The repeatability and inherent high stiffness of this configuration are important for superposition testing methods.

2.1.4 MicroScribe. The MicroScribe G2L digitizer, developed by Immersion Corp. (San Jose, CA), provides spatial information on the rotopod, external load sensor, and the cadaver specimen for use by the application software. Once the relative locations of these components are determined, this software performs all three-dimensional transformations necessary to execute motion and calculates loading response in clinically relevant coordinate systems. One limitation of the MicroScribe (Table 4) is that the resolution and accuracy are not on the same order of magnitude as that of the rotopod. However, since the MicroScribe is used to define the relative coordinate systems of the musculoskeletal simulator components and the specimen, it must also be considered that the variation and precision in determining anatomical references are much larger than the uncertainty in the MicroScribe. For these reasons, the software contains mitigation techniques such as optimization in the foot experiments and hybrid (force and position) control in the knee experiments.

2.1.5 External Sensor Data Acquisition System. The standalone data acquisition system is synchronized with the musculoskeletal simulator, via the common digital synchronization bus and Ethernet, to provide up to 16 additional channels of analog data. Bone or soft tissue strain, joint pressure, or other analog voltage signals are acquired and conditioned using a National Instruments (Austin, TX) PCI-6229 data acquisition board and SCXI-1000 signal conditioning chassis with a SCXI-1143 Butterworth $200 \mathrm{~Hz}$ low-pass, anti-aliasing filter.

2.1.6 Force Platform. A Bertec (Columbus, $\mathrm{OH}$ ) force plate (model 4060) and amplifier (model 6800) were used for the foot experiments in combination with the National Instruments PCI$6034 \mathrm{E}$ data acquisition board for analog/digital conversion of the voltage analog outputs of forces $\left(F_{x}, F_{y}\right.$, and $\left.F_{z}\right)$ and moments $\left(M_{x}, M_{y}\right.$, and $\left.M_{z}\right)$. Characteristics of the force platform are provided in Table 5.

2.1.7 Specimen Mounting Device. An aluminum tube that contains the potted specimen (foot, knee, etc.) slides into a receptacle device, where it is clamped into a stationary position during loading.

2.1.8 Tendon Freeze Clamps. Freeze clamps of two different sizes were developed at the Cleveland Clinic to attach the tendons to the tendon actuator cables. The bodies of these clamps allow the attachment of liquid nitrogen feed lines (Fig. 2).

2.1.9 Tendon Load Cells. Three Omega (Stamford, CT) LCFD-100 load cells (range: 0-445 N, accuracy: $\pm 0.15 \%$ full scale, FS, repeatability: $\pm 0.05 \%$ FS) and one LCFD-500 load cell (range: $0-2224 \mathrm{~N}$, accuracy: $\pm 0.2 \% \mathrm{FS}$, repeatability: $\pm 0.1 \% \mathrm{FS}$ ) were used to measure the force of the individual tendons. Load cells were located in-line between the tendon freeze clamps and tendon actuator cables. In addition, one custom-made load cell incorporated into the pulley of the rotary tendon actuator, manu- 
Table 5 Bertec force platform performance characteristics

\begin{tabular}{ll}
\hline \hline Feature & Value \\
\hline Load rating & $F_{x}, F_{y}: 5000 \mathrm{~N}, F_{z}: 10,000 \mathrm{~N}$ \\
& $M_{x}: 1500 \mathrm{~N} \mathrm{~m}, M_{y}: 1000 \mathrm{~N} \mathrm{~m}, M_{z}: 750 \mathrm{~N} \mathrm{~m}$ \\
Sensitivity & $F_{x}, F_{y}: 0.44 \mathrm{~N} / \mathrm{mV}, F_{z}: 0.89 \mathrm{~N} / \mathrm{mV}$ \\
& $M_{x}: 0.27 \mathrm{~N} \mathrm{~m} / \mathrm{mV}, M_{y}: 0.18 \mathrm{~N} \mathrm{~m} / \mathrm{mV}, M_{z}: 0.13 \mathrm{~N} \mathrm{~m} / \mathrm{mV}$ \\
Linearity & $\pm 2.0 \% \mathrm{FS}$ \\
Hysteresis & $\pm 2.0 \% \mathrm{FS}$ \\
Gain, selectable per channel & $1,2,510,20,50,100$ \\
\hline \hline
\end{tabular}

factured by Strainsert (West Conshohocken, PA), is capable of measuring force in the range of 0-6720 $\mathrm{N}$ (accuracy: $\pm 1 \% \mathrm{FS}$, repeatability: $\pm 0.15 \%$ FS).

2.1.10 Six-Axis Load Cell. The ATI Industrial Automation (Apex, NC) Theta-series SI-1500-240 six-axis load cell (Table 6) was used during knee experiments to measure the loads observed at the tibia attributable to the rotopod. In this configuration, the tibia is purposely mounted in the inverted stationary position.

2.1.11 Knee Flexion Fixture. Given the range of motion of the rotopod, the musculoskeletal simulator is not able to explore the full range of motion of the knee without an additional fixture to provide a seventh DOF. Although relatively small dynamic changes in flexion (about $\pm 10 \mathrm{deg}$ ) are possible with the musculoskeletal simulator, the custom fixture illustrated in Fig. 3 allows for flexion of the knee from $0 \mathrm{deg}$ to $120 \mathrm{deg}$.

2.1.12 Application Software. A software framework for the musculoskeletal simulator has been developed using National Instruments (Austin, TX) LabVIEWTM version 8.2. The framework was tested with both foot and knee applications. The system block diagram (Fig. 4) provides a general organization of application

Table 6 ATI Theta SI-1500-240 load cell performance characteristics

\begin{tabular}{lcccccc}
\hline \hline & \multicolumn{7}{c}{ Value } \\
\cline { 2 - 7 } Feature & $F_{x}$ & $F_{y}$ & $F_{z}$ & $M_{x}$ & $M_{y}$ & $M_{z}$ \\
\hline Load rating (N, N m) & 1500 & 1500 & 3750 & 240 & 240 & 240 \\
Resolution (N, N m) & 0.5 & 0.5 & 1.1 & 0.07 & 0.07 & 0.07 \\
Accuracy (\% FS) & 1.50 & 1.25 & 0.75 & 1.25 & 1.00 & 1.50 \\
\hline \hline
\end{tabular}

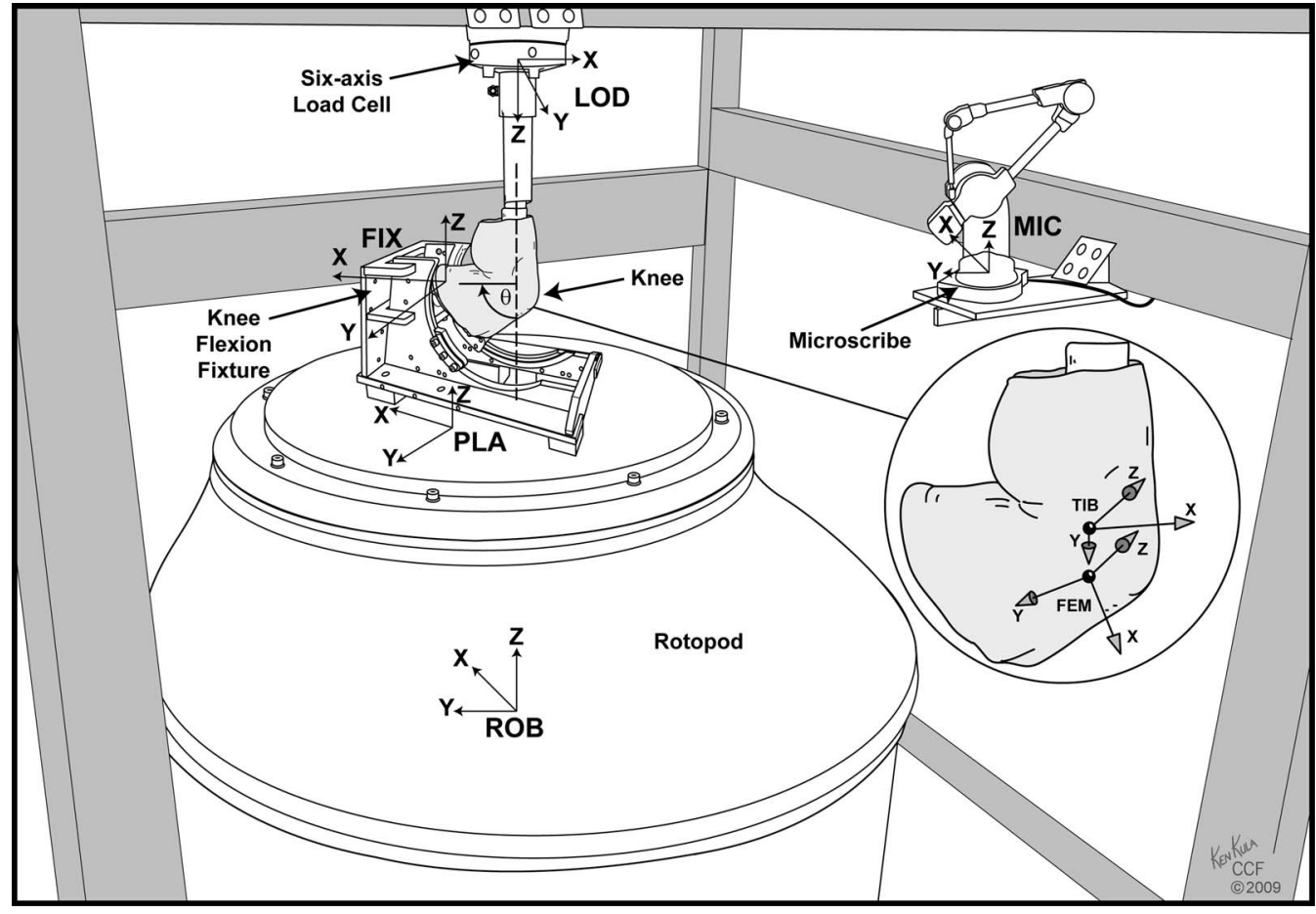

Fig. 3 Simplified illustration of the musculoskeletal simulator, as it would be configured for a knee study. The various coordinate systems shown illustrate the necessary mathematical transformations required to achieve motion of the knee fixture to cause knee flexion (FEM: femur; FIX: knee flexion fixture; LOD: six-axis load cell; MIC: MicroScribe; PLA: rotopod platform; ROB: rotopod base). Reprinted with permission, Cleveland Clinic Center for Medical Art \& Photography @2009. All Rights Reserved. 


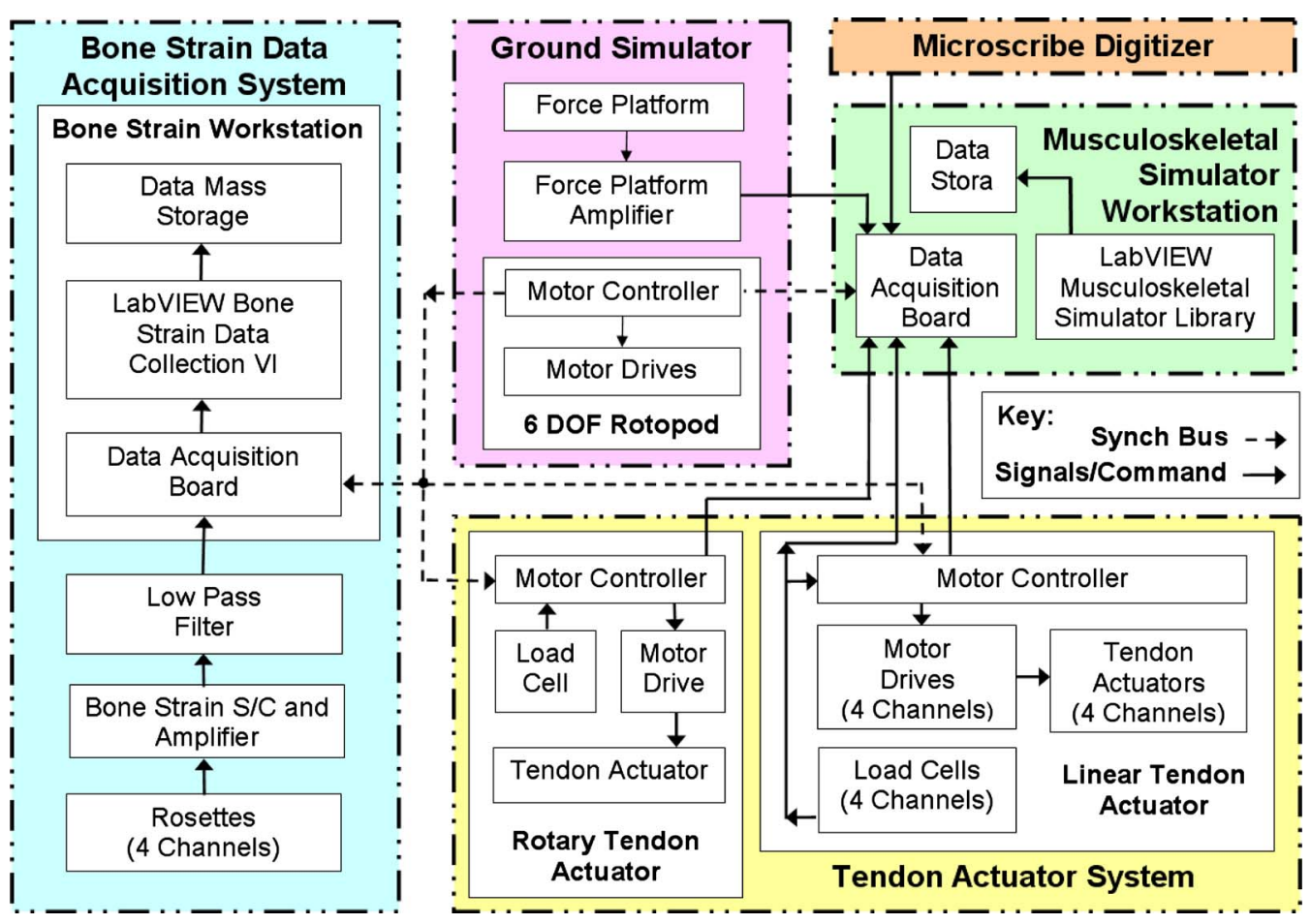

Fig. 4 Musculoskeletal simulator block diagram showing general components required for foot experiments. The synch bus allows synchronization between the rotopod, strain gauge data acquisition, and tendon actuators during simulated gait (DOF, degrees of freedom)-

software required for the foot experiment. The external sensor data acquisition system software has been designed to run on a stand-alone workstation to handle the data acquisition processing, independent of the musculoskeletal simulator workstation processor that provides the main application software. This architecture supports operation in master-slave configuration, by which the musculoskeletal simulator application software controls timing aspects of the external sensor data acquisition system during the experiment.

A graphical user interface captures key aspects of the configuration and setup prior to execution of the experiment simulations. The application software provides the ability to interface with the MicroScribe to digitize the unique anatomical features of each specimen prior to testing to ensure that data are collected in a clinically relevant anatomical coordinate system. A flexible textfile-based system facilitates the input of muscle electromyogram data, kinematic data (motion analysis), and externally induced load data, such as would result from exercise. These input data are used to establish motion trajectories and tendon force profiles in the same clinically relevant coordinate systems as those used for the simulated exercises. During the experiment, the musculoskeletal simulator software produces real-time graphs of engineering data retrieved through analog data input channels. For instance, displays of real-time force and moment data are provided in the tibial coordinate system during knee experiments.

\subsection{Equipment Configuration}

2.2.1 Foot Test Configuration. To conduct foot experiments, the musculoskeletal simulator uses kinetic trajectories (force profiles) for the tendon actuators and for the target ground reaction forces (GRFs). The kinematic trajectory of the tibia relative to the ground, as measured in a gait laboratory, drives the rotopod motion. The musculoskeletal simulator uses iterative optimization techniques to produce the target loading conditions, GRFs, and/or tendon actuators. The anatomical coordinate system is based on a proposed International Society of Biomechanics standard [13].
However, because of the unique nature of cadaveric simulators, a custom reference frame was defined as the tibial coordinate system (TIB). Since the TIB defines the ankle center and is used to orient GRF and ground tibia position data, one needs to consider the orientation of the tibia as well as the foot. Like the knee joint, variations from the standard coordinate system account for missing anatomical reference points caused by the cutting and mounting of limbs. The tibial intercondylar point is replaced with the centroid of the tibia measured at the most proximal location possible, and to increase repeatability of the specimen coordinate system, the mediolateral axis is redefined as an axis perpendicular to the midline of the foot [14]. For orientation of the tibia relative to the ground, Yeadon's [15] "somersault-tilt-twist" variables are used. The Yeadon rotation sequence twist (which is renamed as internal rotation) is measured about the tibial long axis; somersault is measured about the global mediolateral axis. To recreate typical foot-ankle motion, the tibia is fixed horizontally on the surrounding frame, and the force plate is mounted vertically on the top of the rotopod platform to create an inverted ground-tibia motion (Fig. 1). This method provides two major benefits. First, it does not require moving the entire tendon actuator system along with the tibia motion during a simulation. Second, the largest foot-ankle rotation (somersault) can be adequately simulated because of the rotopod's unique ability to provide large rotations in the horizontal plane. One limitation of this configuration is that the inertial loading of the specimen cannot fully be replicated because of the quasi-static nature of the simulations; we compensate for this factor by slight changes in rotopod motion via the optimization process.

2.2.2 Knee Test Configuration. The musculoskeletal simulator, configured to conduct knee experiments, can operate in position or force control. Given a kinematic input file, the musculoskeletal simulator can step through the motion sequence and store data at each position. Given a kinetic input file, the musculoskeletal simulator can ramp to each loading condition via a real-time hybrid controller (simultaneous position and force control). The knee 
joint coordinate system translations and rotations follow the system proposed by Pennock and Clark [16], with one difference: The long axes of femur and tibia do not have the proximal femoral head and ankle joint as reference points since the ends of these bones have been removed to mount the specimen. Instead, these points are replaced with the centroids of the remaining bone at the proximal femur and distal tibia. Although the motions are defined in the knee joint coordinate system, the loads are measured in the tibial reference frame [17]. As a result, the tibia is attached to the load cell since this configuration ensures that the coordinate transformation is a static rather than a dynamic matrix. The load cell is attached to the frame rather than the rotopod, not only to keep the elements clean but also to remove inertial loads and eliminate concerns about wire pinching. The mounting of the knee and flexion fixture are done so as to maximize the joint range of motion with respect to the rotopod range of motion.

\subsection{Data File Organization}

2.3.1 Data File Overview. The rotopod trajectory and servomotor actuator force profiles are defined through a set of data files to provide maximum flexibility and ease of configuration. The data file inputs that must be supplied to define the loading conditions include:

(1) kinematic trajectory (single- or multi-axis)

(a) rotopod motion trajectory of joint or external load device (e.g., force platform simulating the ground, or superposition testing in knee joint coordinate system)

(2) kinetic trajectories (single- or multi-axis)

(a) target load response (e.g., target superior GRF, knee force profile)

(b) individual tendon actuator force profiles

2.3.2 Kinematic Trajectory Data File. The rotopod motion trajectory file contains the trajectory for the relative motion between the joint under investigation and the external loading device during a specified loading condition. The rotopod trajectory is generated through a series of transformations (see Appendix) based on the motion specified in the trajectory data file. In the foot experiment, this file would be the trajectory of the force platform (ground) with respect to the mounted foot. The motion trajectory terms need to be normalized using foot length (FL) and foot width (FW) since these are the characteristic measurements that provide insight to the overall foot size. Time is normalized to a percentage of the total motion time. Before any normalization calculations occur, the raw data (i.e., data collected in the actual gait laboratory) must be transformed to the ground tibia position reference frame, which includes the trajectory variables $(a, m, s, r, t$, and $o$ ) defined below. Additionally, the origin is defined as the point of intersection of the ground plane and the long axis of the tibia at the time when that axis is in the global frontal plane. For physiological normalization, researchers would typically normalize using equations such as

$$
\begin{aligned}
& a=[\text { Anterior translation position } / \mathrm{FL}] \times 100 \% \\
& m=[\text { Medial translation position } / \mathrm{FW}] \times 100 \%
\end{aligned}
$$

$s=\{$ Superior translation position $/[1 / 2 \times(\mathrm{FW}+\mathrm{FL})]\} \times 100 \%$

Time $=[$ Elapsed time/total motion time $] \times 100 \%$

The angles $r, t$, and $o$ do not require scaling

$r=$ Twist angle (positive for internal rotation of the tibia)

$t=$ Tilt angle(positive for lateral tilt) $o=$ Somersault angle(positive for forward rotation)

2.3.3 Target Load Response Data File. This file contains the expected reaction forces and parameters derived from the moments that result from the specific loading condition performed. In the foot experiment, the data would be the expected GRF profiles $\left(F_{a}, F_{m}\right.$, and $\left.F_{s}\right)$ as measured in the gait laboratory, along with the calculated center of pressure $(\mathrm{COP})$ in the anterior $\left(\mathrm{COP}_{a}\right)$ and medial $\left(\mathrm{COP}_{m}\right)$ directions and the internal rotation couple moment $\left(T_{r}\right)$ at the COP. Ultimately, these parameter values should be observed between the foot and the force platform during the simulated walking conditions. As in the case of the external load device motion trajectory, the profile values at any time need to be normalized to physiological parameters and placed into the external loading device coordinate system reference using a standard transformation matrix.

For the foot, this normalization would typically adjust for bodyweight $(\mathrm{BW}), \mathrm{FL}$, and FW. For the COP parameters $\left(\mathrm{COP}_{a}\right.$ and $\mathrm{COP}_{m}$ ), the method used for the averaging and normalization is similar to the method developed by Motriuk and Nigg [18]. Normalization of the forces $\left(F_{a}, F_{m}\right.$, and $\left.F_{s}\right)$ makes use of the commonly accepted practice of using percentage of BW (\% BW). The last parameter $T_{r}$ is scaled by percentage of $\mathrm{BW}$ and the average of FL and FW. The target force platform response data file would include the following normalized parameters at each normalized time profile point:

$$
F_{a}=[\text { Measured force in the anterior axis } / \mathrm{BW}] \times 100 \%
$$

$F_{m}=[$ Measured force in the medial axis/BW $] \times 100 \%$

$F_{s}=[$ Measured force in the superior axis $/ \mathrm{BW}] \times 100 \%$

$$
\begin{aligned}
& \mathrm{COP}_{a}=[\mathrm{COP} \text { in the anterior axis/FL }] \times 100 \% \\
& \mathrm{COP}_{m}=[\mathrm{COP} \text { in the medial axis/FW }] \times 100 \%
\end{aligned}
$$

$T_{r}=\{$ Couple moment in the internal rotation axis $/[\mathrm{BW} \times 1 / 2$

$$
\times(\mathrm{FW}+\mathrm{FL})]\} \times 100 \%
$$

2.3.4 Tendon Actuator Force Profile Data Files. The application expects that the tendon force profile during the simulated loading will be provided in terms of normalized force at each normalized time as defined below:

$$
\text { Force }=[\text { Actuator force } / \mathrm{BW}] \times 100 \%
$$

\subsection{Force Control Techniques}

2.4.1 Iterative Optimization. After any experiment simulation, optimization can be used to adjust the input data file for the external load environment/joint motion trajectory (i.e., results in an adjusted rotopod trajectory) and individual tendon actuator force profiles to eliminate offset between the actual and target load responses. The optimization algorithm used in the foot experiment can calculate optimized rotopod trajectories and tendon actuator force profiles based on actual data recorded from a previous experiment and the target GRF. For example, an experiment would be conducted to simulate the stance phase of walking, and then the experimenters would look at the results to determine what optimization modes are necessary. The optimization feature is used to iteratively make the necessary adjustments until convergence criteria are achieved.

The optimization algorithm is a combination of individual configurable pseudo-fuzzy logic controllers. Each controller uses one input and one output. The input signal is the error in one of the six 
GRF channels, and the output signal is then added to the chosen simulator channel (e.g., superior motion, tibialis anterior force, etc.). The controller processes the input by selective windowing (\% stance range within which data are to be analyzed), applying the chosen algorithm (i.e., use mean, absolute value, or point-bypoint), low-pass filtering, multiplying by a gain parameter, and finally adding to the output channel data from the previous run to produce the optimized output signal for that same channel. Multiple controllers acting on the same simulator channel are collectively summed to produce the optimized trajectories used for the subsequent test.

Optimization of muscle forces is considered to be adaptive such that the viscoelastic response of the tendon from the previous experiment is taken into consideration when making adjustments for the subsequent experiment. For instance, if the superior GRF $\left(F_{s}\right)$ did not achieve the target peak value at toe-off (e.g., the triceps surae muscle group did not reach the target tension at that time), then optimization can increase the force to this muscle group at that same time by an amount equal to the following:

$$
\begin{aligned}
F_{\text {triceps surae }}(\text { new })= & F_{\text {triceps surae }}(\text { previous })+\text { Gain } \times\left(F_{s \text { target }}\right. \\
& \left.-F_{s \text { actual }}\right)
\end{aligned}
$$

Similarly, optimization provides the flexibility necessary to adjust for positional misalignment between the joint coordinate system and device contacting the joint to provide loading. To illustrate this possibility, consider the origin of the tibia coordinate system $X, Y$, and $Z$ in the ankle (identified as TIB in Fig. 1). If the actual origin were $1 \mathrm{~mm}$ in the $Z$-direction from what was recorded with the MicroScribe during set up of the experiment, then it would manifest itself as low $F_{s}$ during the experiment, and optimization can be invoked to adjust for this discrepancy. The result would be to shift the force platform trajectory by a constant amount in the $Z$-direction for all time increments during simulated stance, such that the $Z$-position (new) is now computed as

$$
\begin{aligned}
Z \text {-position }(\text { new })= & Z \text {-position }(\text { previous })+\text { Gain } \times \operatorname{Mean}\left(F_{s}\right. \text { target } \\
& \left.-F_{s \text { actual }}\right)
\end{aligned}
$$

In this case, the mean value is computed for the difference in $F_{S}$ across all time increments. This mean is then multiplied by a constant gain value to achieve the $Z$-value offset for the force platform trajectory.

2.4.2 Real-Time Hybrid Control. In the knee experiments, the aim is to provide simultaneous position and force control. The flexion axis of the knee has very little stiffness, and controlling moment about that axis would be unlikely to provide a unique solution. For this reason, the joint is controlled in three axes of force control (anterior, medial, and superior), two axes of torque control (varus and internal rotations), and one axis of angle control (flexion). This PID hybrid control scheme operates in a variation in the knee joint coordinate system to maximize decoupling. The controller transforms the data from the load cell coordinate system to the tibial coordinate system [19]. Then superior force and varus torque are decoupled into two superior forces, each located at the center of each femoral condyle. Following the PID algorithm, the resulting command signals are integrated with respect to time, recoupled to the knee joint coordinate system, and transformed to the rotopod coordinate system. In addition, the hybrid controller employs other tools, such as gain scheduling and feed forward, to further enhance speed and stability.

2.5 Validation Methods. Validation of this complex system included evaluating the general capabilities of the major components (subsystems) as well as demonstrating the performance of the full system when configured to conduct foot and knee experiments.
2.5.1 Tendon Actuator Accuracy and Repeatability Validation Tests. A foot study designed to simulate gait was used to test the mean absolute accuracy and repeatability of the tendon actuators at achieving the target tendon force levels. Six experiments conducted on two specimens provided data from multiple experiments at the same loading conditions. Absolute errors were computed between actual and target force at each time interval during stance for each experiment and reported as a mean \pm 1 standard deviation. Repeatability was visualized by plotting the target force against the actual force for various experiments for periods of simulated muscle contractions. Simulated relaxation was not included in the plots because hysteresis that results between contraction and relaxation further complicates the plots (i.e., two points per experiment at each stance point).

2.5.2 Component Synchronization Validation Tests. To synchronize the entire system, the low-level programs of the rotopod and tendon actuators, and the internal and external data acquisition systems were coded to start their respective processes at the moment when the rotopod's controller generates a digital falling trigger signal. Since the external data acquisition system was coded to poll the digital trigger signal every $1 \mathrm{~ms}$, the timing delay between the digital trigger signal and the external data was a maximum of $1 \mathrm{~ms}$. The internal data acquisition system preacquires data and is postprocessed to align to the trigger, resulting in a delay, which is also $\leq 1 \mathrm{~ms}$. The timing delay of the mechanical components' motion from the digital trigger signal was evaluated by performing a step functionlike motion profile. Ten tests each were conducted on the rotopod, rotary tendon actuator, and linear tendon actuators to measure the motion delay from the start of the synchronization trigger signal. System synchronization accuracy can be estimated by the following equation:

$$
\begin{aligned}
& \text { Synchronization system accuracy } \\
& =\frac{\text { Max. delay }+ \text { Min. delay }}{2} \pm \frac{\text { Max. delay }- \text { Min. delay }}{2}
\end{aligned}
$$

2.5.3 Rotopod Position Accuracy Validation Test. The rotopod provides motion, force input, or both to the joint of interest. The control of force is done through real-time feedback control, as in the knee experiments, or iterative force control, as in the foot experiments. Fundamentally, position is iterated to reach the target force. Therefore, a series of tests were run to determine the quasistatic and dynamic translational accuracy of the rotopod when loaded (with a payload of $98.2 \mathrm{~kg}$ ) and unloaded. The quasi-static test motion path was a stepped triangle wave (10 mm per step) over the full range of motion ( $\pm 100 \mathrm{~mm}$ in each axis), quantifying uniaxial position error. The dynamic test path was a $0.167 \mathrm{~Hz}$ sinusoidal waveform corresponding to a peak speed of $100 \mathrm{~mm} / \mathrm{s}$ (maximum capability of the rotopod) for the same range of motion. A Heidenhain Corp. (Shaumburg, IL) model LS679 linear encoder, having an accuracy of $10 \mu \mathrm{m}$ and a resolution of $0.5 \mu \mathrm{m}$, was used to measure the movement of the robot. Accuracy was assessed by maximum $(\max )$ and root-mean-square (rms) positional errors for the full range of motion (similar to the foot experiment) and for the center range of motion $( \pm 30 \mathrm{~mm}$, as in the knee experiment).

2.5.4 Optimization Validation Test. Experiment optimization was invoked to target the heel strike and the latter half of stance during foot experiments to achieve reasonable simulated walking. This capability was tested through a series of seven experiments: Experiments 1-4 focused on adjusting offsets during heel strike, whereas experiments 5-7 focused on adjusting the muscle forces from midstance through toe-off.

2.5.5 Foot Test Demonstration. The foot experiment configuration of the musculoskeletal simulator has been used to measure various biomechanical parameters in studies of normal and patho- 

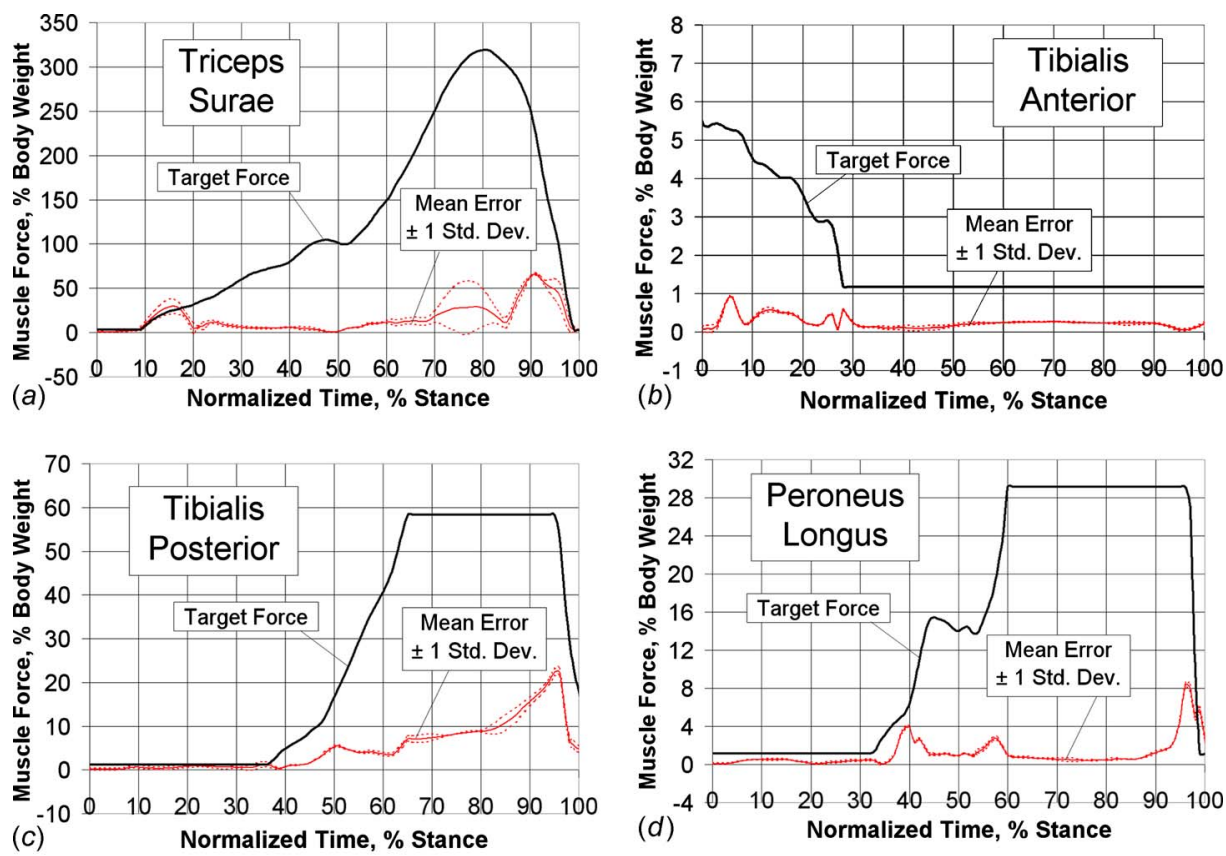

$(d)$

Normalized Time, \% Stance

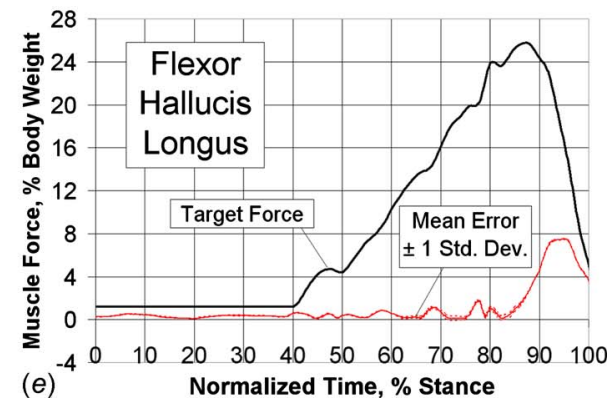

Fig. 5 Tendon actuator accuracy results for two experiments of three runs each, in which under closed-loop feedback, the actuator of the musculoskeletal simulator simulates muscle contractions. Muscles included (a) triceps surae, (b) tibialis anterior, (c) tibialis posterior, (d) peroneus longus, and (e) flexor hallucis longus. Note that absolute error is shown as a mean \pm 1 standard deviation. Target force is included as a reference.

logical gaits. In a recent study [20], it was used to investigate the effects of diabetes on the midfoot joint pressures. A foot study designed to acquire tibial and calcaneal bone strain data during simulating gait is used to demonstrate the musculoskeletal simulator capabilities in a foot experiment configuration. Tibial and calcaneal strain data were collected using Vishay MicroMeasurements (Raleigh, NC) rosette C2A-06-031WW-120. Testing was performed to verify that analog data (in this case, strain data) could be synchronized through the digital synchronization bus and collected during the entire stance phase of simulated walking in a reliable and repeatable manner. Two 2100 system signal conditioning amplifiers (Vishay Micro-Measurements) were used to provide quarter-bridge circuit conditioning and amplification required for these strain gauge rosettes. The locations of these rosettes were anterior tibia (lateral and medial sides), posterior tibia, and lateral calcaneus for a total of 12 channels of raw strain data. The foot study simulated walking at one-fourth speed and varying BW percentages $(16.5 \%, 38.4 \%, 66.7 \%$, and $100 \%$ BW). Graphs of the target and actual GRF data, along with the tendon force data, for a representative experiment are presented.

2.5.6 Knee Test Demonstration. The musculoskeletal simulator has been used to study native kinematics, arthroplasty, and surgical techniques in the knee joint. In one study, the knee test system was programmed to apply 108 combinations of the following loading conditions at three flexion angles $(0 \mathrm{deg}, 30 \mathrm{deg}$, and $60 \mathrm{deg})$ : internal/external rotation $(0 \mathrm{~N} \mathrm{~m}, \pm 5 \mathrm{~N} \mathrm{~m})$, varus/valgus $(0 \mathrm{~N} \mathrm{~m}, \pm 10 \mathrm{~N} \mathrm{~m})$, compression $(100 \mathrm{~N}, 700 \mathrm{~N})$, and posterior drawer $(0 \mathrm{~N}, 100 \mathrm{~N})$. The combined loading condition was ramped, held, and released in $2 \mathrm{~s}, 3 \mathrm{~s}$, and $1 \mathrm{~s}$, respectively. The error between the target and actual forces, or torques, is analyzed continuously as well as during the plateau (at which point auxiliary data is typically collected).

\section{Results}

3.1 Tendon Actuator Accuracy and Repeatability Results. Tests conducted to measure the error between target and actual tendon actuator forces revealed a large variability in absolute error (which was dependent on the stance time; Fig. 5), but these tests demonstrated that within multiple runs of the same experiment there was excellent repeatability (Fig. 6).

3.2 Component Synchronization Results. Test results of synchronization revealed that the rotopod contributes the largest delay at $10.8 \pm 1.0 \mathrm{~ms}$, followed by the linear actuator at $5.2 \pm 1.4 \mathrm{~ms}$, then the rotary actuator at $4.1 \pm 1.0 \mathrm{~ms}$. Using Eq. (14), the total synchronization system accuracy was $6.7 \pm 2.0 \mathrm{~ms}$. 

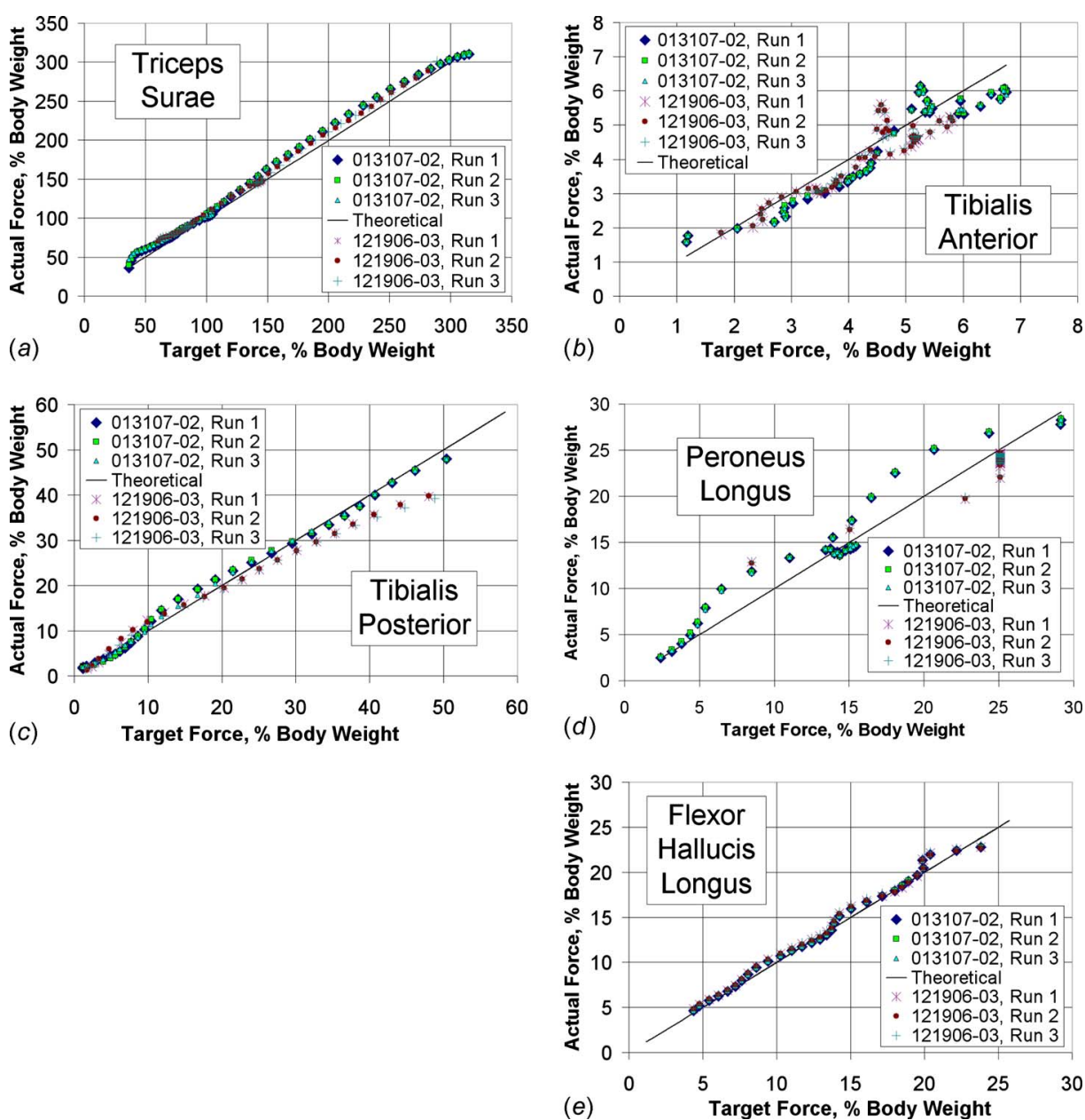

Fig. 6 Tendon actuator repeatability results for two experiments of three runs each, in which under closed-loop feedback, the actuator of the musculoskeletal simulator simulates muscle contractions. Muscles included (a) triceps surae, $(b)$ tibialis anterior, $(c)$ tibialis posterior, (d) peroneus longus, and (e) flexor hallucis longus. Note that relative accuracy can be seen in deviation from the theoretical line.

3.3 Rotopod Positioning Results. The rotopod positioning test results (Table 7) ranged from $10 \mu \mathrm{m}$ to $359 \mu \mathrm{m}$, depending on measurement condition. The $Z$-axis position error is roughly 2 times the error for the $X$ - and $Y$-axes. In general, loaded errors were higher than the unloaded errors by $1.2,1.3$, and 1.6 times, for the $X-, Y$-, and $Z$-axis, respectively.

3.4 Optimization Results. A typical optimization scenario is depicted in Fig. 7. Experiments 1-4 were used to adjust the superior GRF to achieve the target level at the initial heel strike con- tact by changing the anterior and superior coordinates of the tibial coordinate system. Table 8 summarizes what changes were made for the first four experiments to simulate heel strike. Experiments 5-7 used time-based adjustments to the plantarflexors (triceps surae, flexor hallucis, tibialis posterior, and peroneus longus) to bring the superior GRF to within $\pm 10 \%$ of the target force during loading response, midstance, terminal stance, and toe-off contact phases.

3.5 Foot Test Demonstration. The optimization target of

Table 7 Rotopod positioning results

\begin{tabular}{lcccccccc}
\hline \hline & \multicolumn{2}{c}{$X$-axis } & & \multicolumn{2}{c}{$Y$-axis } & & \multicolumn{2}{c}{$Z$-axis } \\
\cline { 2 - 3 } Position error $(\mu \mathrm{m})$ & Unloaded & Loaded & & Unloaded & Loaded & & Unloaded & Loaded \\
\hline Quasi-static full (max) & 56 & 99 & & 37 & 50 & & 74 & 234 \\
Quasi-static full (rms) & 24 & 28 & & 18 & 29 & & 36 & 84 \\
Quasi-static center (max) & 55 & 27 & & 32 & 44 & & 58 & 61 \\
Quasi-static center (rms) & 26 & 16 & & 19 & 24 & & 33 & 30 \\
Dynamic full (max) & 89 & 108 & & 79 & 127 & & 206 & 359 \\
Dynamic full (rms) & 31 & 38 & & 30 & 31 & & 63 & 110 \\
Dynamic center (max) & 27 & 39 & & 62 & 58 & & 95 & 85 \\
Dynamic center (rms) & 10 & 15 & & 26 & 19 & & 52 & 49 \\
\hline \hline
\end{tabular}

Max: maximum; rms: root-mean-square. 


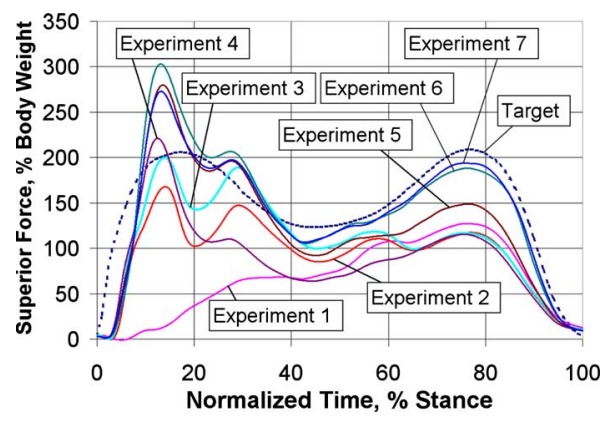

Fig. 7 Optimization results for seven experiments, showing convergence of superior force against the target toe-off region profile during simulated gait using the musculoskeletal simulator. $\pm 10 \%$ was achieved at heel strike and toe-off in the superior axis during simulated gait using the musculoskeletal simulator (Fig. 8). In the anterior and COP channels, the goal was to optimize the kinetic and kinematic trajectories to the point where the target and actual curves had a similar form. For this experiment, further optimization to better achieve the target profiles was not necessary to obtain the desired bone strain results.

3.6 Knee Test Demonstration. The hybrid controller demonstrated that low errors can be achieved on the superior compression channel during the course of the 108 combined loading conditions (see Fig. 9 for a representative graph). The highest errors (rms and max) were found to be in the continuous comparison analysis (Table 9).

\section{Discussion}

4.1 Tendon Actuator Accuracy and Repeatability. Force accuracy results achieved with the tendon actuators during the

Table 8 Optimization during heel strike

\begin{tabular}{|c|c|c|c|}
\hline $\begin{array}{l}\text { Experiment } \\
\text { No. }\end{array}$ & $\begin{array}{l}\text { Anterior } \\
\text { offset } \\
(\mathrm{mm})\end{array}$ & $\begin{array}{l}\text { Superior } \\
\text { offset } \\
(\mathrm{mm})\end{array}$ & Summary of results \\
\hline 1 & 0 & -13 & Starting point; no heel contact with force platform \\
\hline 2 & 4 & -11 & $\begin{array}{l}\text { Force platform contacted the heel } 4 \mathrm{~mm} \text { forward (anterior direction) } \\
\text { of the initial run and moved } 2 \mathrm{~mm} \text { closer (superior direction) to the } \\
\text { bottom of the foot. This achieved } 36 \% \mathrm{BW} \text { (target } 44 \% \mathrm{BW} \text { ). }\end{array}$ \\
\hline 3 & 5 & -10.5 & $\begin{array}{l}\text { Force platform trajectory was adjusted another } 1 \mathrm{~mm} \text { and closer to } \\
\text { the mounted foot by } 0.5 \mathrm{~mm} \text {. This achieved } 43 \% \mathrm{BW} \text {. }\end{array}$ \\
\hline 4 & 9.5 & -10.5 & $\begin{array}{l}\text { Force platform trajectory was adjusted } 4.5 \mathrm{~mm} \text { forward (anteriorly) } \\
\text { from previous run with no change in the proximity to the foot } \\
\text { (superior direction) at the start. This had an adverse affect by } \\
\text { overshooting to } 47 \% \text { BW. Note: The previous iteration's anterior } \\
\text { offset }(5 \mathrm{~mm} \text { ) was ultimately used for the final experiment settings. }\end{array}$ \\
\hline
\end{tabular}

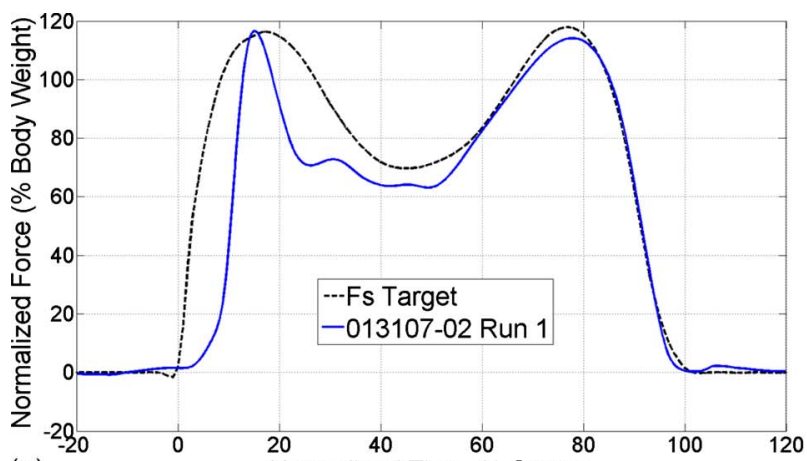

(a) Normalized Time, \% Stance

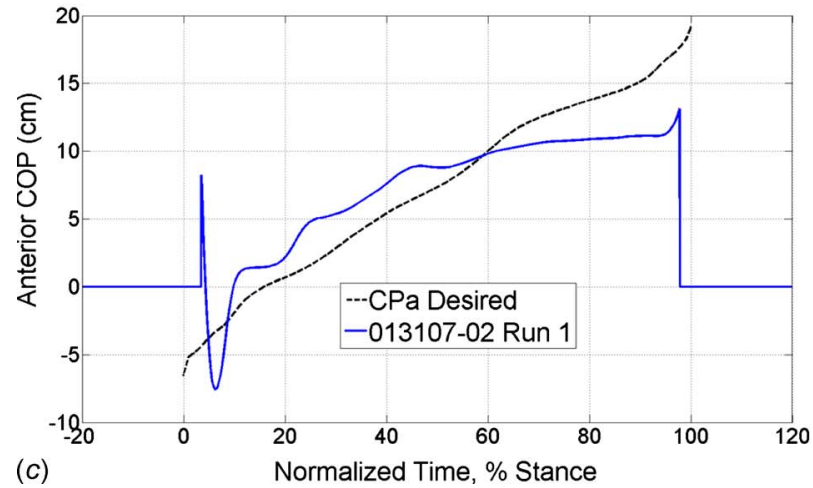

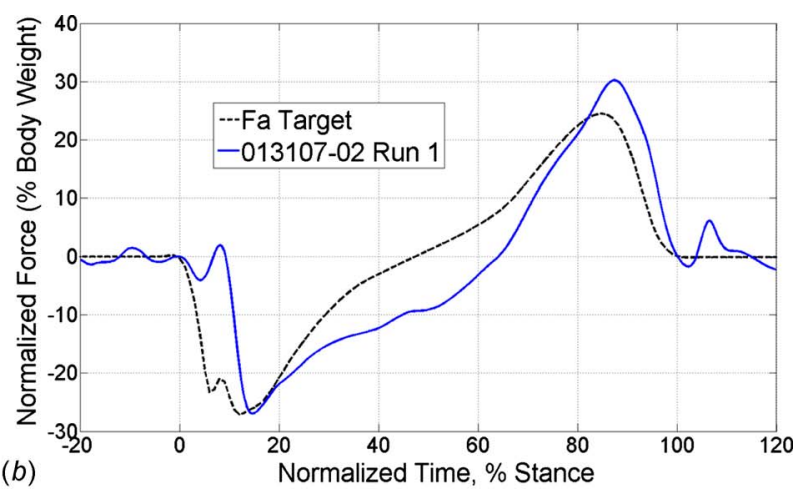

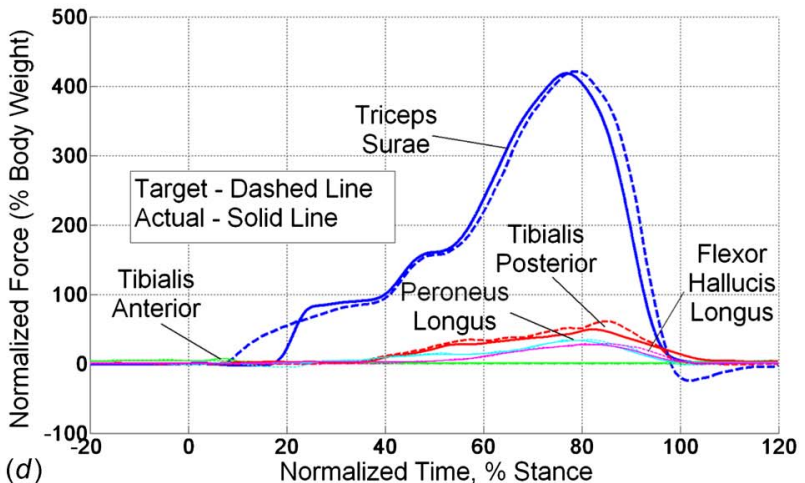

Fig. 8 Selected results from the foot bone strain study using the musculoskeletal simulator are shown. Full-physiological loading is demonstrated through (a) the superior and $(b)$ anterior ground reaction forces, (c) anterior center of pressure, and (d) muscle forces. Results shown are indicative of a typical experiment run. 


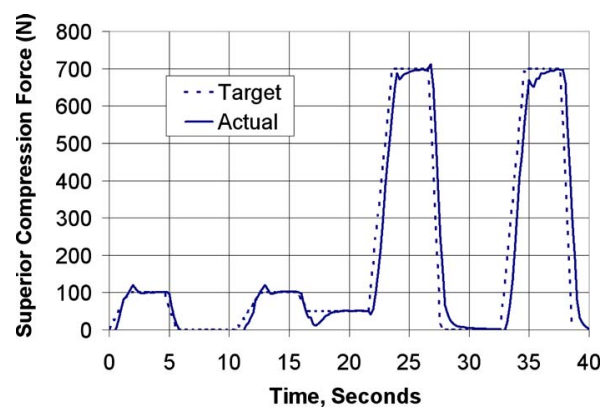

Fig. 9 Representative superior compression force profile of the real-time proportional-integral-derivative (PID) hybrid control for a knee experiment using the musculoskeletal simulator.

musculoskeletal simulator performance verification process were sufficient to accurately simulate gait for the foot bone strain study. The ability of the tendon actuators to achieve the target muscle force profile is dependent on the resolution of the in-line load cells and controller gains (PID). The load cell resolution was found to correlate $\left(R^{2}=0.85\right)$ with the tendon actuator accuracy. The load cell used with the actuator simulating the tibialis posterior muscle had a resolution of $0.54 \mathrm{~N}$ per count (12 bit analog/digital converter counts), the load cell used with the triceps surae actuator had a resolution of $0.19 \mathrm{~N}$ per count, and the remaining load cells had resolutions of $0.10 \mathrm{~N}$ per count. Excellent repeatability results were demonstrated for the tendon actuators, with an average error of $0.3 \% \mathrm{BW}$. Tendon actuator accuracy posed no limitations to the particular foot study; therefore, no further optimization was deemed necessary. A one-time adjustment was made to the controller PID gains, velocity parameters, and acceleration parameters for the linear tendon actuators. This adjustment resulted in a substantial performance improvement, which was sufficient for the foot study. Future studies that require an even higher level of accuracy may achieve it by optimization of these parameters.

4.2 Component Synchronization. Provided that the duration of the activity being simulated is significantly larger than the synchronization error $(6.7 \pm 2.0 \mathrm{~ms})$, the effect of the error will be insignificant for future researchers. For the foot study presented, the simulated walking motion was $2.8 \mathrm{~s}$. Therefore, this error represents $0.24 \%$ of the total experiment time and is not considered significant.

4.3 Rotopod Positioning Discussion. The highest error values measured were for $Z$-axis motion, potentially due to considerable changes in the configuration of the robot legs. Loading generally increased error magnitude but was not pronounced for the center range of motion. The error values were less than those found in other studies, [10] and therefore, are adequate for in vitro reproduction of certain motions.

4.4 Optimization. A typical optimization procedure was discussed, showing that the system has the necessary flexibility to successfully optimize the trajectory (required for heel strike adjustment) and for muscle force optimization (required for the latter phase of stance). During the foot study, it was found that typically within 3-6 iterations of trajectory optimization, it was possible to obtain a heel strike force within the target limit of $\pm 10 \%$ of the target superior GRF. Similarly, within 4-8 iterations of muscle force optimization, the latter half of stance was within this limit. Optimization adjusted the target muscle forces by an amount proportional to the measured parameter (superior force error); therefore, subsequent iterations of optimization converged on acceptable muscle forces regardless of whether or not they matched the target force set point. Stability of the optimization algorithm is therefore much more dependent on repeatability of the actuators and the rotopod, which has been shown to be very high. Although the fuzzy logic controllers were effective on this experiment, one limitation is that the algorithms provided nonunique solutions to the optimization, given that there were six inputs (GRF) and 11 outputs (6DOF kinematics and five tendon actuators). Future enhancement of the optimization algorithm may be necessary, depending on the requirements for a given study. To provide for this possibility, the musculoskeletal simulator software can be customized within the existing software framework to allow the implementation of fuzzy logic, model predictive, linear optimization, or any other control philosophy.

4.5 Foot and Knee Test Demonstrations. Through the completion of the performance validation process, several key features of the musculoskeletal simulator have been demonstrated. Multiple joints have undergone 6DOF simulations at fullphysiological loading conditions. Full-physiological loadings of the foot and knee were achieved with the musculoskeletal simulator in a stable and highly repeatable manner.

Foot experiments used programmable loading conditions and operated at one-fourth walking speed. Synchronization of system components, accuracy of tendon actuators and of rotopod position, and the results of the foot experiment systematically demonstrate that the musculoskeletal simulator is able to simulate an entire gait cycle through coordinated motion of the rotopod and tendon actuators while simultaneously recording 12 channels of bone strain.

In the knee experiment, one limitation to achieving the dynamic motion demonstrated by the foot experiment is the static adjustability of the flexion fixture. As a result of this limitation, tests had to be paused in order to manually adjust the fixture to provide greater changes in knee flexion. Work has recently been completed to remove this constraint by developing a rotary stage mounted on top of the rotopod. This stage provides dynamic flexion capabilities for knee, shoulder, and hip experiments with a range of $\pm 180 \mathrm{deg}$.

The representative errors in the real-time hybrid control are minimal in the plateau measurements and sufficient for testing where quasi-static combinations of loads are applied. Figure 9 suggests that the continuous errors in Table 9 result from the inherent lag in PID control algorithms. In studies for which realtime dynamic loading is desired, improvements would need to be made in the response time of the control system by modifying this algorithm or implementing a new one.

Table 9 Representative knee force/torque control errors

\begin{tabular}{lccccc}
\hline \hline & \multicolumn{5}{c}{ Value } \\
\cline { 2 - 6 } Force/torque control error & $\begin{array}{c}F_{\text {lateral }} \\
(\mathrm{N})\end{array}$ & $\begin{array}{c}F_{\text {anterior }} \\
(\mathrm{N})\end{array}$ & $\begin{array}{c}F_{\text {superior }} \\
(\mathrm{N})\end{array}$ & $\begin{array}{c}T_{\text {Varus }} \\
(\mathrm{N} \mathrm{m})\end{array}$ & $\begin{array}{r}T_{\text {ER }} \\
(\mathrm{N} \mathrm{m})\end{array}$ \\
\hline Plateau (max) & 1 & 3 & 10 & 0.1 & 0.2 \\
Plateau (rms) & $<1$ & 1 & 4 & 0.04 & 0.1 \\
Continuous (max) & 73 & 69 & 330 & 9.4 & 1.4 \\
Continuous (rms) & 11 & 16 & 71 & 1.3 & 0.3 \\
\hline \hline
\end{tabular}

$T_{\mathrm{ER}}$ : torque, external rotation; max: maximum; rms: root-mean-square. 


\section{Conclusions}

The musculoskeletal simulator has been shown to simulate the biomechanics of human motion through (i) a set of actuators that, when connected to selected tendons traversing a joint, can imitate muscular contractions, and (ii) a rotopod that can simulate environmentally induced loading of and contact with the cadaver specimen. The benefit of these coupled systems is that they enable fully synchronized joint loading at physiological levels, at or near real-time speeds. The design of the musculoskeletal simulator makes it readily adaptable for investigation of many different joint systems. The musculoskeletal simulator has been developed to enable fundamental research that is focused on injury prevention, but the applications extend into other areas such as the evaluation of surgical interventions and total joint replacements and the development of rehabilitation regimens.

\section{Acknowledgment}

The authors would like to acknowledge the support for this research from NASA Grant No. NNJ05HF55G and from the Cleveland Clinic Musculoskeletal Core Center, funded in part by NIAMS Core Center Grant No. 1P30 AR-050953. In addition, the efforts of Katherine Muterspaugh in providing LabVIEW routines for sampling and storing bone strain data are appreciated. Special thanks to Ken Kula (Cleveland Clinic Center for Medical Art and Photography) for providing the artist renditions of the Musculoskeletal Simulator. Finally, the support of the Cleveland Clinic is gratefully acknowledged through the contributions of Peter R. Cavanagh, Ph.D., D.Sc. (now of the University of Washington/ Seattle) for providing the rotopod and Ahmet Erdemir, Ph.D., from the Computational Biomodeling Core, for providing rotopod test results.

\section{Appendix: Transformation of Three-Dimensional Kine- matic Data to Rotopod Trajectory (Foot and Knee Ex- amples)}

This appendix illustrates the kinematic chain equation, as shown in Eqs. (15) and (16), for typical foot and knee experiments, respectively. The expressions include reference frames for the rotopod base (ROB), the rotopod platform (PLA), the force plate (GND), the knee flexion fixture (FIX), the six-axis load cell (LOD), the MicroScribe (MIC), the tibia (TIB), and the femur (FEM). The static transformation matrices for the foot are $\mathbf{T}_{\mathrm{ROB}, \mathrm{MIC}}, \mathbf{T}_{\mathrm{PLA}, \mathrm{GND}}$, and $\mathbf{T}_{\mathrm{TIB}, \mathrm{MIC}}$. The corresponding dynamic matrices are $\mathbf{T}_{\mathrm{ROB}, \mathrm{PLA}}$ and $\mathbf{T}_{\mathrm{GND}, \mathrm{TIB}}$. The static transformation matrices for the knee are $\mathbf{T}_{\mathrm{ROB}, \mathrm{MIC}}, \mathbf{T}_{\mathrm{TIB}, \mathrm{MIC}}$, and the configurable $\mathbf{T}_{\text {PLA,FIX }}$. The corresponding dynamic matrices are $\mathbf{T}_{\mathrm{ROB}, \mathrm{PLA}}$ and $\mathbf{T}_{\mathrm{FEM}, \mathrm{TIB}}$. These equations can be used to derive the elements of any one dynamic matrix given the other dynamic matrix (such as deriving rotopod positions given the motion of the tibia relative to the ground) that may have been collected in a gait laboratory setting. Refer to Figs. 1 and 3 for the location of each reference frame.

$$
\mathbf{T}_{\mathrm{ROB}, \mathrm{MIC}}=\mathbf{T}_{\mathrm{ROB}, \mathrm{PLA}}(q) \cdot \mathbf{T}_{\mathrm{PLA}, \mathrm{GND}} \cdot \mathbf{T}_{\mathrm{GND}, \mathrm{TBD}} \cdot \mathbf{T}_{\mathrm{TIB}, \mathrm{MIC}}(r)
$$

$$
\begin{aligned}
\mathbf{T}_{\mathrm{ROB}, \mathrm{MIC}}= & \mathbf{T}_{\mathrm{ROB}, \mathrm{PLA}}(q) \cdot \mathbf{T}_{\mathrm{PLA}, \mathrm{FIX}}(\theta) \cdot \mathbf{T}_{\mathrm{FIX}, \mathrm{FEM}} \\
& \cdot \mathbf{T}_{\mathrm{FEM}, \mathrm{TIB}}\left(K_{\mathrm{JCS}}\right) \cdot \mathbf{T}_{\mathrm{TIB}, \mathrm{MIC}}
\end{aligned}
$$

where the rotopod coordinates are as follows:

$$
q=(x, y, z, \text { roll, pitch, yaw })
$$

the ground/tibia position are as follows:

$$
r=(a, m, s, r, t, o)
$$

the flexion fixture setting are as follows:

$$
\theta=\text { Nominal knee flexion angle }
$$

the knee joint coordinates $[16,21]$ are as follows:

$$
K_{\mathrm{JCS}}=(a, b, c, \alpha, \beta, \gamma)
$$

\section{References}

[1] Lanyon, L. E., Hampson, W. G., Goodship, A. E., and Shah, J. S., 1975, "Bone Deformation Recorded In Vivo From Strain Gauges Attached to the Human Tibial Shaft," Acta Orthop. Scand., 46, pp. 256-268.

[2] Burr, D. B., Milgrom, C., Fyhrie, D., Forwood, M., Nyska, M., Finestone, A., Hoshaw, S., Saiag, E., and Simkin, A., 1996, "In Vivo Measurement of Human Tibial Strains during Vigorous Activity," Bone (N.Y.), 18, pp. 405-410.

[3] Weiss, J. A., Gardiner, J. C., Ellis, B. J., Lujan, T. J., and Phatak, N. S., 2005, "Three-Dimensional Finite Element Modeling of Ligaments: Technical Aspects," Med. Eng. Phys., 27, pp. 845-861.

[4] Sharkey, N. A., and Hamel, A. J., 1998, "A Dynamic Cadaver Model of the Stance Phase of Gait: Performance Characteristics and Kinetic Validation," Clin. Biomech. (Bristol, Avon), 13, pp. 420-433.

[5] Milgrom, C., Finestone, A., Hamel, A., Mandes, V., Burr, D., and Sharkey, N., 2004, "A Comparison of Bone Strain Measurements at Anatomically Relevant Sites Using Surface Gauges Versus Strain Gauged Bone Staples," J. Biomech., 37, pp. 947-952.

[6] Hurschler, C., Emmerich, J., and Wülker, N., 2003, "In Vitro Simulation of Stance Phase Gait—Part I: Model Verification,” Foot Ankle Int., 24, pp. 614622.

[7] Kim, K.-J., Kitaoka, H. B., Luo, Z.-P., Ozeki, S., Berglund, L. J., Kaufman, K. R., and An, K.-N., 2001, "In Vitro Simulation of the Stance Phase in Human Gait," Journal of Musculoskeletal Research, 5, pp. 113-121.

[8] Kim, K. J., Uchiyama, E., Kitaoka, H. B., and An, K. N., 2003, "An In Vitro Study of Individual Ankle Muscle Actions on the Center of Pressure," Gait Posture, 17, pp. 125-131.

[9] Ward, E. D., Smith, K. M., Cocheba, J. R., Patterson, P. E., and Phillips, R. D., 2003, "In Vivo Forces in the Plantar Fascia During the Stance Phase of Gait: Sequential Release of the Plantar Fascia," J. Am. Podiatr. Med. Assoc., 93, pp. 429-442.

[10] Howard, R. A., Rosvold, J. M., Darcy, S. P., Corr, D. T., Shrive, N. G., Tapper, J. E., Ronsky, J. L., Beveridge, J. E., Marchuk, L. L., and Frank, C. B., 2007, "Reproduction of In Vivo Motion Using a Parallel Robot," ASME J. Biomech. Eng., 129, pp. 743-749.

[11] Cavanagh, P. R., ed., 1990, Biomechanics of Distance Running, Human Kinetics Publishers, Champaign, IL, pp. 92-93.

[12] Riley, P. O., Dicharry, J., Franz, J., Croce, U. D., Wilder, R. P., and Kerrigan, D. C., 2008, "A Kinematics and Kinetic Comparison of Overground and Treadmill Running," Med. Sci. Sports Exercise, 40, pp. 1093-1100.

[13] Wu, G., Siegler, S., Allard, P., Kirtley, C., Leardini, A., Rosenbaum, D., Whittle, M., D'Lima, D. D., Cristofolini, L., Witte, H., Schmid, O., Stokes, I., and Standardization and Terminology Committee of the International Society of Biomechanics, 2002, "ISB Recommendation on Definitions of Joint Coordinate System of Various Joints for the Reporting of Human Joint MotionPart I: Ankle, Hip, and Spine," J. Biomech., 35, pp. 543-548.

[14] Isman, R. E., and Inman, V. T., 1969, "Anthropometric Studies of the Human Foot and Ankle,” Bull. Prosthet. Res., 11, pp. 97-129.

[15] Yeadon, M. R., 1990, "The Simulation of Aerial Movement-I. The Determination of Orientation Angles From Film Data," J. Biomech., 23, pp. 59-66.

[16] Pennock, G. R., and Clark, K. J., 1990, “An Anatomy-Based Coordinate System for the Description of the Kinematic Displacements in the Human Knee," J. Biomech., 23, pp. 1209-1218.

[17] Woo, S. L., Kanamori, A., Zeminski, J., Yagi, M., Papageorgiou, C., and Fu, F. H., 2002, "The Effectiveness of Reconstruction of the Anterior Cruciate Ligament With Hamstrings and Patellar Tendon. A Cadaveric Study Comparing Anterior Tibial and Rotational Loads," J. Bone Jt. Surg., Am. Vol., 84-A, pp. 907-914.

[18] Motriuk, H. U., and Nigg, B. M., 1990, "A Technique for Normalizing Centre of Pressure Paths," J. Biomech., 23, pp. 927-932.

[19] Fujie, H., Livesay, G. A., Woo, S. L., Kashiwaguchi, S., and Blomstrom, G., 1995, "The Use of a Universal Force-Moment Sensor to Determine In-Situ Forces in Ligaments: A New Methodology," ASME J. Biomech. Eng., 117, pp. $1-7$.

[20] Lee, D. G., and Davis, B. L., 2009, "Assessment of the Effects of Diabetes on Midfott Joint Pressure Using a Robotic Gait Simulator," Foot Ankle Int., 30, pp. 767-772.

[21] Grood, E. S., and Suntay, W. J., 1983, "A Joint Coordinate System for the Clinical Description of Three-Dimensional Motions: Application to the Knee," ASME J. Biomech. Eng., 105, pp. 136-144. 